\title{
A Smart Thermal Environment Monitor Based on IEEE 1451.2 Standard for Global Networking
}

\author{
Yanfeng Wang, Member, IEEE, Makoto Nishikawa, Ryuichi Maeda, Masaichi Fukunaga, and \\ Kenzo Watanabe, Fellow, IEEE
}

Abstract-A temperature and relative humidity (RH) monitor using a thermistor and a polyimide-film RH Sensor is developed for global assessment of thermal environments. The smart transducer interface module includes the relaxation oscillators for signal conditioning and a one-chip 16-bit microcomputer for networking. The microcomputer accommodates the calibration tables as well as the mandatory transducer electronic data sheets specified by the IEEE 1451.2 standard. The online calibration using the calibration table and the ratiometric signal conditioning allow $\pm 0.14{ }^{\circ} \mathrm{C}$ accuracy over the temperature range from $-20^{\circ} \mathrm{C}$ to $50{ }^{\circ} \mathrm{C}$ and $\pm 2.5 \% \mathrm{RH}$ accuracy over the $\mathrm{RH}$ range from $20 \% \mathrm{RH}$ to $90 \% \mathrm{RH}$. Beside these high-accuracy measurements using low-cost sensors, the monitor features an adaptive architecture for global networking.

Index Terms-Energy saving, environment, measurement, networking, relative humidity, smart interface, temperature.

\section{INTRODUCTION}

I NCREASING energy consumption is accelerating environmental pollution. The global warming and the atmospheric air pollution due to $\mathrm{CO}_{2}$ and exhaust gases are typical phenomena. Under such circumstances, some countries specify wholesome working and living environments in a building and mandate the continuous environment monitoring. Along with the environment accessment, energy saving is highly requested to reduce the pollution. According to statistics in advanced countries, $40 \%$ of the electric energy required for maintaining office environments is consumed by air conditioning. It follows, therefore, that localized control of an air conditioner depending on a thermal map in an office space is one of the most effective approaches to the energy saving. The thermal mapping requires distributed monitors which measure local temperature and relative humidity $(\mathrm{RH})$.

With the above-mentioned applications in mind, a thermal environment monitor is developed which measures temperature and $\mathrm{RH}$ in an indoor space [1]. The design strategics are low cost, high accuracy, easy installation, and easy networking for distributed measurements. For low-cost implementation, a thermistor and a polyimide-film RH sensor are used. To achieve high-accuracy measurement with these low-cost sensors, ratiometric signal processing and online calibration are introduced. For easy installation into a wall-embedded outlet box, simple

Manuscript received June 15, 2003; revised July 7, 2004.

Y. Wang and K. Watanabe are with the Research Institute of Electronics, Shizuoka University, Hamamatsu 432-8011, Japan.

M. Nishikawa, R. Maeda, and M. Fukunaga are with the Systems Development Promotion Department, Matsushita Electric Works, Osaka 571-8686, Japan.

Digital Object Identifier 10.1109/TIM.2004.839767

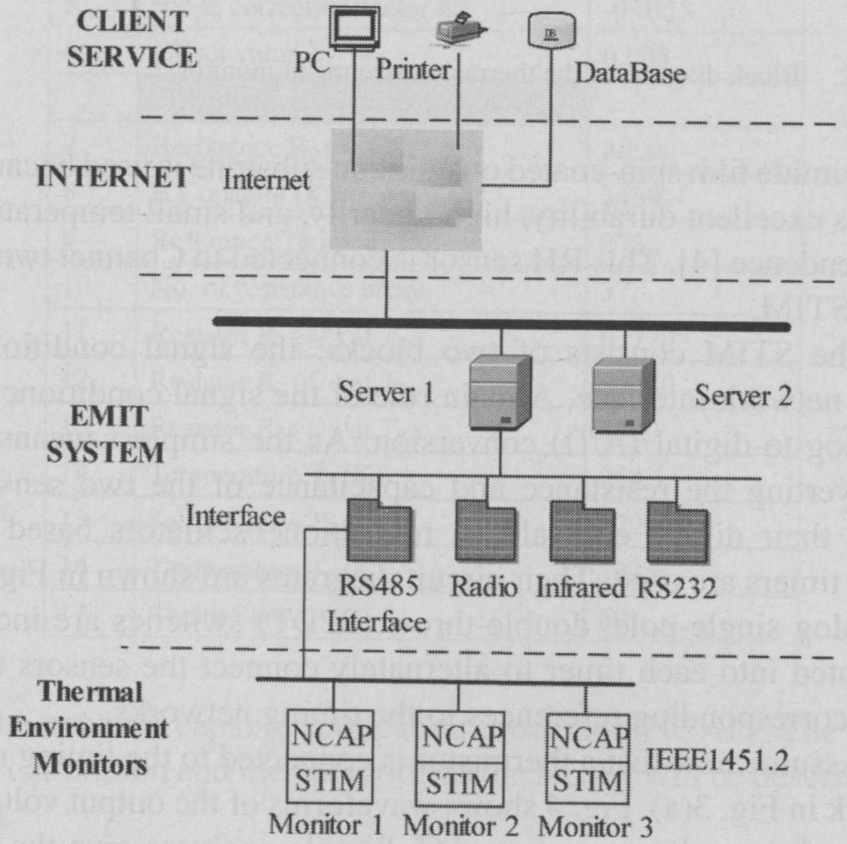

Fig. 1. Network structure of thermal environment monitoring.

circuits and a one-chip microcomputer are used for signal processing and communication interface. For easy networking, the hierarchical architecture is adopted and a smart interface is designed based on IEEE1451.2 standard [2], [3]. These details will be described in the following sections.

\section{ARCHITECTURE}

Fig. 1 shows the hierarchical architecture of the distributed thermal environment monitor. The lowest level is the thermal monitor consisting of the sensors and the smart transducer interface module (STIM) defined in IEEE1451.2 standard. The intermediate level is the embedded micro internet technology (EMIT) system which serves as a bridge between the thermal monitor and the wide or local area network which lies in the upper level. A user positions the highest level and can access the thermal monitor through the Internet bya user-friendly web page or other software. Hardware and software shown in the architecture will be described in the following section.

\section{A. Smart Transducer Interface Module}

Fig. 2 shows a schematic diagram of the thermal environment monitor. It is composed of sensors and the STIM for signal conditioning and networking. For low-cost implementation, a negative temperature coefficient (NTC) thermistor is used for temperature measurement and connected to Channel one of the STIM. For RH measurement, a capacitive sensor consisting of a 


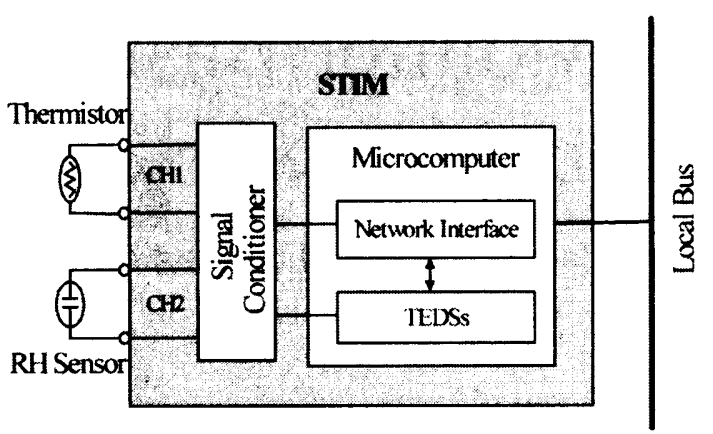

Fig. 2. Block diagram of the thermal environment monitor.

polyimide film spin-coated on a silicon substrate is used because of its excellent durability, high linearity, and small temperature dependence [4]. This RH sensor is connected to Channel two of the STIM.

The STIM consists of two blocks: the signal conditioner and network interface. A main role of the signal conditioner is analog-to-digital (A/D) conversion. As the simplest means of converting the resistance and capacitance of the two sensors into their digital equivalents, relaxation oscillators based on 555 timers are used. Their circuit diagrams are shown in Fig. 3. Analog single-pole, double-throw (SPDT) switches are incorporated into each timer to alternately connect the sensors and the corresponding references to the timing networks.

Assume now that a thermistor is connected to the timing network in Fig. 3(a). Fig. 4 shows waveforms of the output voltage $v_{0}$ and the voltage $v_{c}$ across $C$. Simple analyses give the expressions for $v_{c}$ in (1) shown at the bottom of the page. $R(T)$ is the thermistor resistance and $C$ is the capacitance of the timing capacitor.

The periods $\tau_{H}$ and $\tau_{L}$ during which $v_{0}$ assumes $V c c$ and 0 , respectively, are then given by

$$
\begin{aligned}
\tau_{H} & =\left(R_{1}+R(T)\right) C \ln 2 \\
\tau_{L} & =R(T) C \ln 2 .
\end{aligned}
$$

The oscillation period $\tau\left(R_{\mathrm{T}}\right)$ when a thermistor is connected to the timing network is thus given by

$$
\tau\left(R_{\mathrm{T}}\right)=\tau_{H}+\tau_{L}=C\left[R_{1}+2 R(T)\right] \cdot \ln 2 .
$$

In deriving (4), the comparators and the switch inside the 555 timer are assumed to be delay-free.

Similarly, the oscillation period $\tau\left(R_{r}\right)$ when the reference resistor $R_{r}$ is connected to the timing network is given by

$$
\tau\left(R_{r}\right)=\left(R_{1}+2 R_{r}\right) C \ln 2 .
$$

Taking the ratio $\gamma_{R}$ between $\tau\left(R_{\mathrm{T}}\right)$ and $\tau\left(R_{r}\right)$, one can measure the resistance $R(T)$ of a thermistor by

$$
R(T)=\frac{1}{2}\left[\left(\gamma_{R}-1\right) R_{1}+2 \gamma_{R} R_{r}\right]
$$

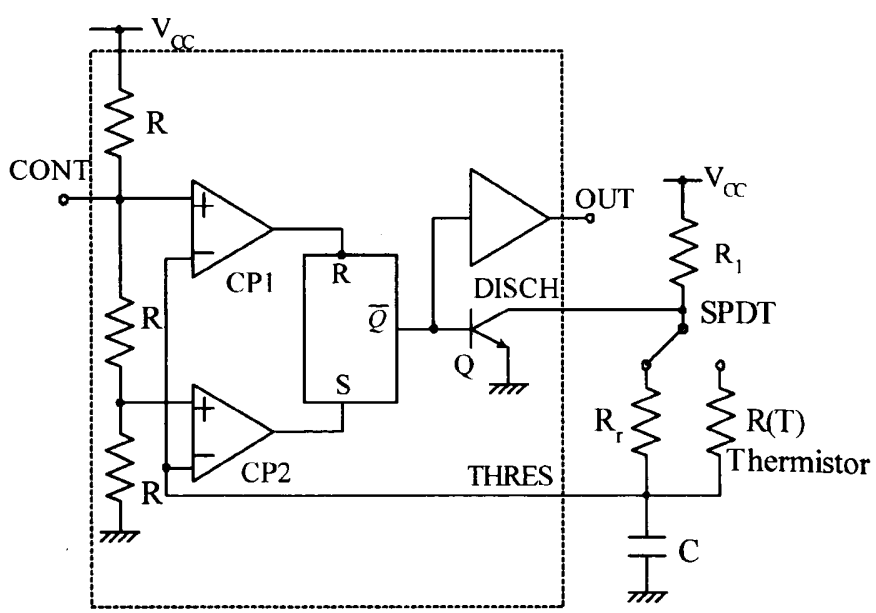

(a)

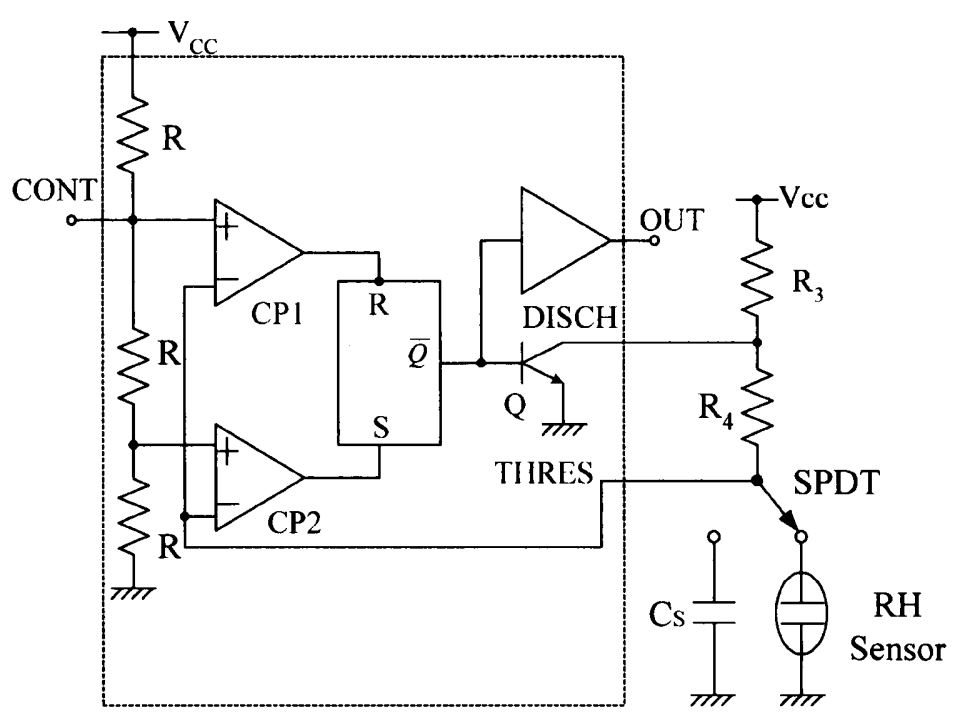

(b)

Fig. 3. Signal conditioners for (a) thermistor and (b) RH sensor.

where

$$
\gamma_{R}=\frac{\tau\left(R_{\mathrm{T}}\right)}{\tau\left(R_{r}\right)}=\frac{R_{1}+2 R(T)}{R_{1}+2 R_{r}} .
$$

Similarly, the capacitance $\mathrm{C}(\% \mathrm{RH})$ of the $\mathrm{RH}$ sensor can be measured by Fig. 3(b) as follows:

$$
C(\% R H)=\gamma_{c} C_{\mathrm{s}}
$$

where

$$
\gamma_{c}=\frac{\tau(\% R H)}{\tau\left(C_{\mathrm{s}}\right)}
$$

is the ratio of the oscillation periods when the RH sensor and the reference capacitor $\mathrm{C}_{\mathrm{s}}$ are connected to the timing network in Fig. 3(b). These ratiometric strategies reduce the effects of uncertainties of timing components other than the sensors, thereby allowing high-accuracy measurements of the resistance and capacitance of the sensors.

$$
v_{c}(t)= \begin{cases}\frac{1}{3} V_{c c}+\left(V_{c c}-\frac{V_{c c}}{3}\right)\left(1-e^{-\left(1 /\left(R_{1}+R(T)\right) C\right) t}\right), & \left(0 \leq t \leq \tau_{H}\right) \\ \frac{2}{3} V_{c c} e^{-(1 / R(T) C)\left(t-\tau_{H}\right)}, & \left(\tau_{H} \leq t \leq \tau_{L}+\tau_{H}\right) .\end{cases}
$$




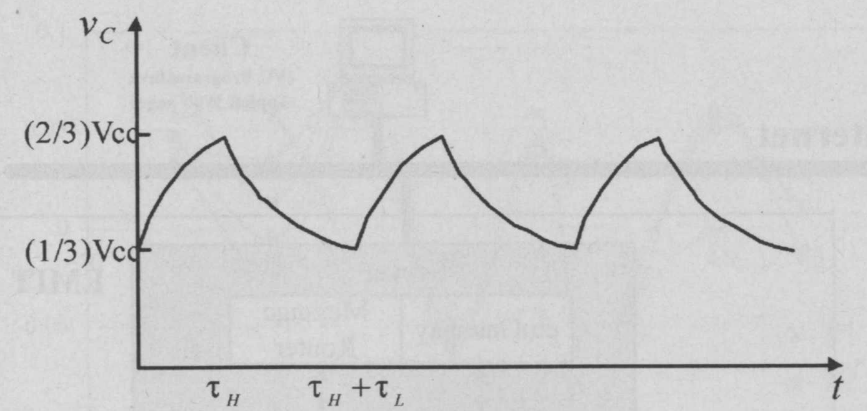

(a)

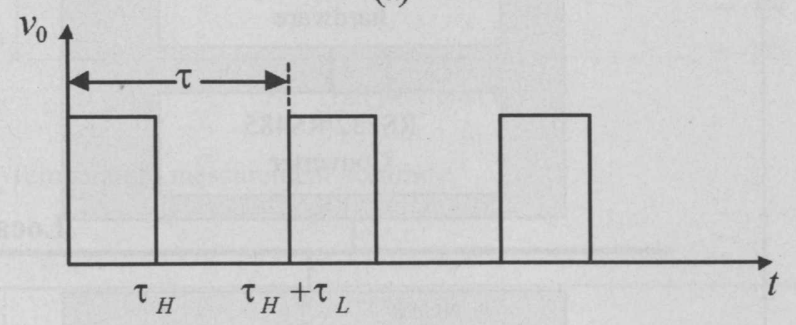

(b)

Fig. 4. Waveforms of (a) output voltage and (b) the voltage across $\mathrm{C}$ of the signal conditioner.

In designing the signal conditioner of the thermistor, the power dissipation should be taken into account. Referring again to Figs. 3(a) and 4, the current $i(t)$ flowing through a thermistor is given by

$i(t$

$= \begin{cases}\frac{V_{c c}-v_{c}(t)}{R_{1}+R_{S}+R(T)} \cong \frac{2}{3} \frac{V_{c c}}{R_{1}+R_{S}+R(T)}\left(1-\frac{t}{2 \tau_{H}}\right), & \left(0 \leq t \leq \tau_{H}\right) \\ -\frac{v_{c}(t)}{R_{S}+R(\tau)} \cong-\frac{2}{3} \frac{V_{c c}}{R_{S}+R(T)}\left(1-\frac{t-\tau_{H}}{2 \tau_{L}}\right), & \left(\tau_{H} \leq t \leq \tau\right) .\end{cases}$

In deriving the previous expressions, a linear approximation of the exponential waveform is used. The power dissipated in a thermistor is then given by

$$
P=R(T) \cdot I_{\mathrm{rms}}^{2}=\frac{7}{12}\left(\frac{2}{3} V_{c c}\right)^{2} \cdot \frac{1}{R_{1}+R(T)}
$$

The increase in the thermistor temperature $\alpha\left({ }^{\circ} \mathrm{C}\right)$ due to the power dissipation is given by

$$
\alpha=\theta \cdot P
$$

where $\theta\left({ }^{\circ} \mathrm{C} / \mathrm{W}\right)$ is the thermal radiation coefficient of a thermistor. This temperature increase decreases in inverse proportion to the timing resistor $R_{1}$, and $R_{1}$ should be selected large enough for $\alpha$ to be smaller than the specified accuracy of temperature measurement.

A 16-bit one-chip microcomputer is used to count the oscillation periods and to obtain temperature and relative humidity by subsequent operations. It also accommodates excellent communications facilities for networking. ${ }^{1}$ Meta, Meta Identification, Channel Identification, and Channel TEDSs defined by IEEE1451.2 standard are stored in the built-in memories. Besides these mandatory TEDSs, the calibration TEDSs are provided for online calibration. The microcomputer also functions
TABLE I

CH1 CALIBRATION TEDS

\begin{tabular}{|l|l|l|}
\hline Field & Description & Data \\
\hline 1 & Table Length & 54 \\
\hline & Signal conditioner sub-block & \\
\hline 2 & Timing Resister $\mathrm{R}_{1}$ & 2000 \\
\hline 3 & Reference Resister $\mathrm{R}_{\mathrm{r}}$ & 47500 \\
\hline 4 & Timing Capacitor $\mathrm{C}$ & 0.018 \\
\hline 5 & Slope correction factor $\beta$ & -0.0118 \\
\hline 6 & Offset value $\delta$ & 0.008 \\
\hline & Thermistor parameter sub-block & \\
\hline 7 & Resistance $\mathrm{R}_{0}(\Omega)$ & 30000 \\
\hline 8 & B Constant $(\mathrm{K})$ & 3450 \\
\hline 9 & Reference $\mathrm{Temp} . \mathrm{T}_{0}(\mathrm{~K})$ & 273 \\
\hline 10 & No. of resistance array & 3 \\
\hline 11 & Resistor $\mathrm{R}_{1}(\Omega)$ at $\mathrm{T}_{1}$ & 67770 \\
\hline 12 & Resistor $\mathrm{R}_{2}(\Omega)$ at $\mathrm{T}_{2}$ & 27280 \\
\hline 13 & Resistor $\mathrm{R}_{3}(\Omega)$ at $\mathrm{T}_{3}$ & 4160 \\
\hline 14 & Temperature $\mathrm{T}_{1}(\mathrm{~K})$ & 254.1 \\
\hline 15 & Temperature $\mathrm{T}_{2}(\mathrm{~K})$ & 272.8 \\
\hline 16 & Temperature $\mathrm{T}_{3}(\mathrm{~K})$ & 322.6 \\
\hline 17 & Check Sum & 69 \\
\hline & & \\
\hline
\end{tabular}

as the network capable application processor (NCAP). The online calibration and the operation as the NCAP will be described next.

\section{B. Calibration}

The thermistor resistance $R(T)$ is related to temperature $T$ as follows

$$
R(T)=R_{o} \exp B\left(\frac{1}{T}-\frac{1}{T_{o}}\right)
$$

where $R_{0}$ is the thermistor resistance at a reference temperature $T_{0}$ and $B$ represents the temperature sensitivity. Once $R(T)$ is known by the ratiometric measurement, as given by (6), we can convert it to temperature $T$ by (13). It should be noted that $\gamma_{R}$ given by (7) is derived assuming the ideal condition. In fact, $\gamma_{R}$ deviates from the ideal value (7) due to response delays of the comparators inside the timer, and the error reaches $1.7 \%$ when $\gamma_{R}=0.5$. According to the sensitivity analysis based on (13), the error less than $0.1 \%$ is required for the temperature measurement accurate to $\pm 0.1^{\circ} \mathrm{C}$ over the range from $-20^{\circ} \mathrm{C}$ to $50{ }^{\circ} \mathrm{C}$. To meet this requirement, the error is approximated by a linear function of the measured ratio $\gamma_{R}$ and thermistor resistance $R(T)$ is derived by (6) in terms of the compensated ratio $\gamma$ given by

$$
\gamma=(1-\beta) \gamma_{R}-\delta
$$

The slope correction factor $\beta$ and the offset $\delta$ are determined $a$ priori by replacing the thermistor with known resistors.

Table I shows the data structure of CH1 Calibration TEDS. Data stored are also shown. The thermistor resistance $R\left(T_{\mathrm{i}}\right)$ at temperature $T_{\mathrm{i}}$ is acquired through a network connected to the thermal environment monitor under calibration in a temperature- and RH-controlled chamber. Using these resistances, the 
TABLE II

CH2 CALIBRATION TEDS

\begin{tabular}{|l|l|l|}
\hline Field & Description & Data \\
\hline 1 & Table Length & 28 \\
\hline & Signal conditioner sub-block & \\
\hline 2 & Timing Resister $\mathrm{R}_{3}(\Omega)$ & 1000000 \\
\hline 3 & Timing Resister $\mathrm{R}_{4}(\Omega)$ & 1000000 \\
\hline 4 & Reference Capacitor $\mathrm{C}_{\mathrm{s}}(\mathrm{pF})$ & 330 \\
\hline & Sensor parameter sub-block & \\
\hline 5 & Offset Capacitance $\mathrm{C}_{0}(\mathrm{pF})$ & 326 \\
\hline 6 & Sensitivity $\mathrm{S}$ & 0.63 \\
\hline 7 & Reference Temp. $\mathrm{T}_{0}(\mathrm{~K})$ & 273 \\
\hline 8 & Temperature Coefficient $k$ & -0.0032 \\
\hline 9 & Check Sum & 95 \\
\hline
\end{tabular}

microcomputer in the STIM calculates the $B$ constant of the thermistor and stores it into the table.

The capacitance $C(\% \mathrm{RH})$ of the $\mathrm{RH}$ sensor is related to relative humidity $\% \mathrm{RH}$ as follows

$$
C(\% R H)=\gamma_{C} C_{S}=C_{0}+S(\% R H-60 \% R H)
$$

where $S$ denotes the sensitivity and $C_{o}$ is the capacitance at $60 \% \mathrm{RH}$. The ratio $\gamma_{C}$ also deviates from the ideal relation (9) due to the response delays of the comparators, but the compensation is not taken into account because the capacitance change with RH is small and the ratio is close to one. The capacitance of the RH sensor changes also with temperature because the permittivity of water depends on temperature. This dependence is compensated by a linear function of temperature

$$
\% R H(T)=\% R H\left\{1-k\left(T-T_{o}\right)\right\}
$$

where $\% \mathrm{RH}$ is the $\mathrm{RH}$ measured by $(15), k$ is the temperature coefficient, $T$ and $T_{0}$ are the measured and reference temperatures, respectively.

Table II shows the data structure of $\mathrm{CH} 2$ Calibration TEDS. Data stored into the sensor parameter sub-block are all acquired by the online calibration.

The STIM under environment monitoring measures the resistance of the thermistor and the capacitance of the $\mathrm{RH}$ sensor with reference to $R_{r}$ and $C_{\mathrm{s}}$, respectively, calculates temperature and RH with reference to Calibration TEDSs, and stores the results into the functional addresses assigned by IEEE 1451.2 standard. Upon request by clients, these data are sent to a server and displayed.

\section{Networking}

Fig. 5 shows the detailed network architecture. The embedded micro internet technology (EMIT) is used to connect the STIM to the Internet. ${ }^{2}$ EMIT functions are divided into two parts: The gateway functions for protocol conversion between internet and a local bus are accommodated into a personal computer as a server. Communication functions such as a network adapter and emMicro routines are accommodated into NCAP. A one-chip, 16-bit microcomputer is also used as NCAP.

\footnotetext{
${ }^{2}$ Available online: www.eware.com
}

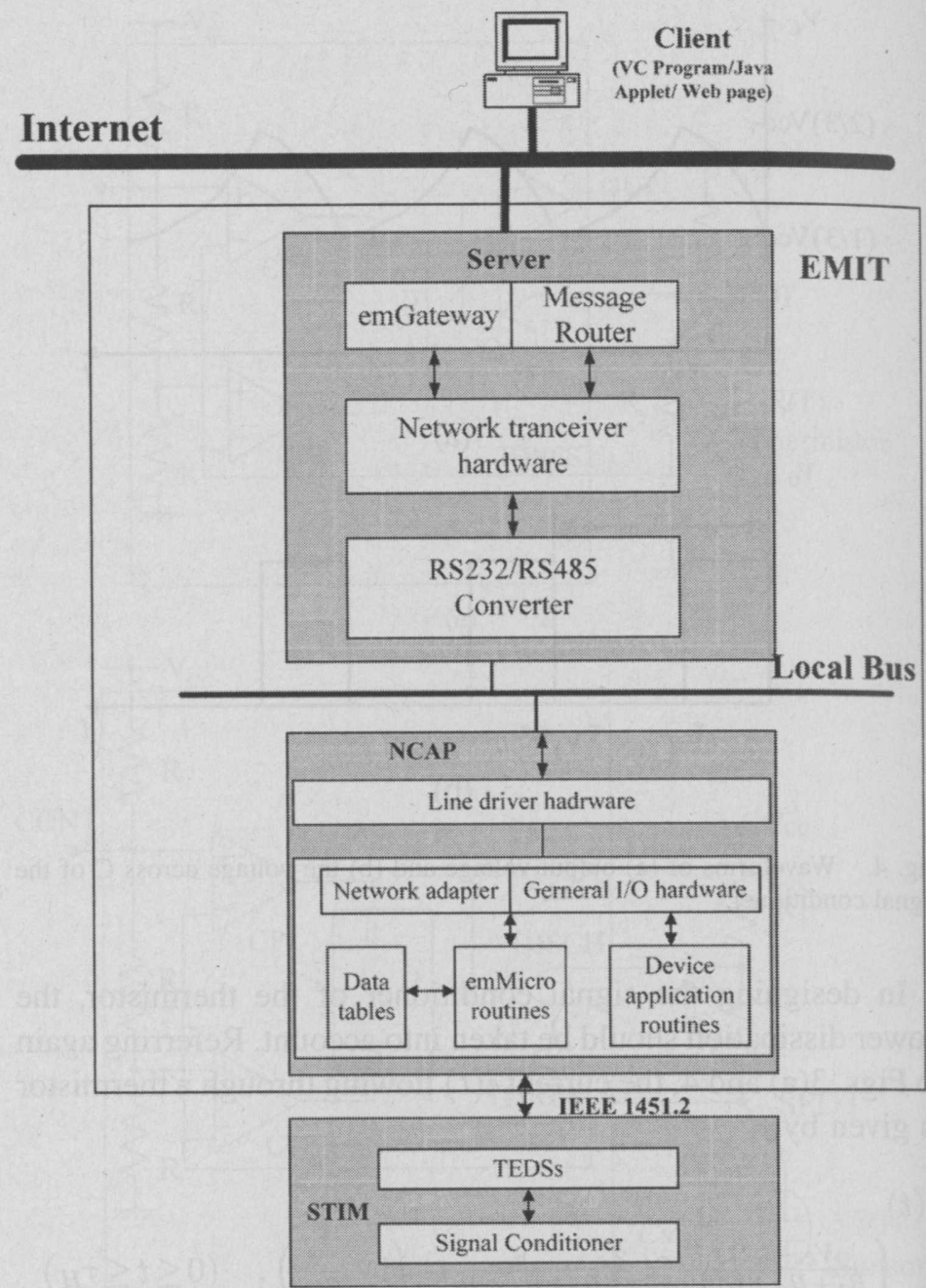

Fig. 5. Network architecture.

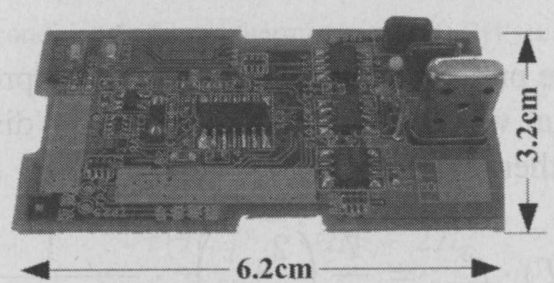

Fig. 6. Prototype STIM.

NCAP is allotted an organizationally unique identifier (OUI), accessed by a server through RS232-to-RS485 converter, and then communicates with STIM. Protocols to access STIM also follow IEEE 1451.2 standard.

This separation of the EMIT functions allows clients, which may be web page, database, or such high-level application softwares as Visual C++ and Java, to easily access the environment monitor using TCP/IP protocols [5], [6].

\section{PROTOTYPE MONITOR}

Fig. 6 shows the STIM of the thermal environment monitor implemented on a printed-circuit (PC) board. The board is designed such that the monitor be housed in a wall-embedded type of an outlet box. A thermistor and a perforated metal package in which the RH sensor is mounted can be seen to the right. The one-chip microcomputer is attached together with network connectors to the other side of the board. The supply voltage 


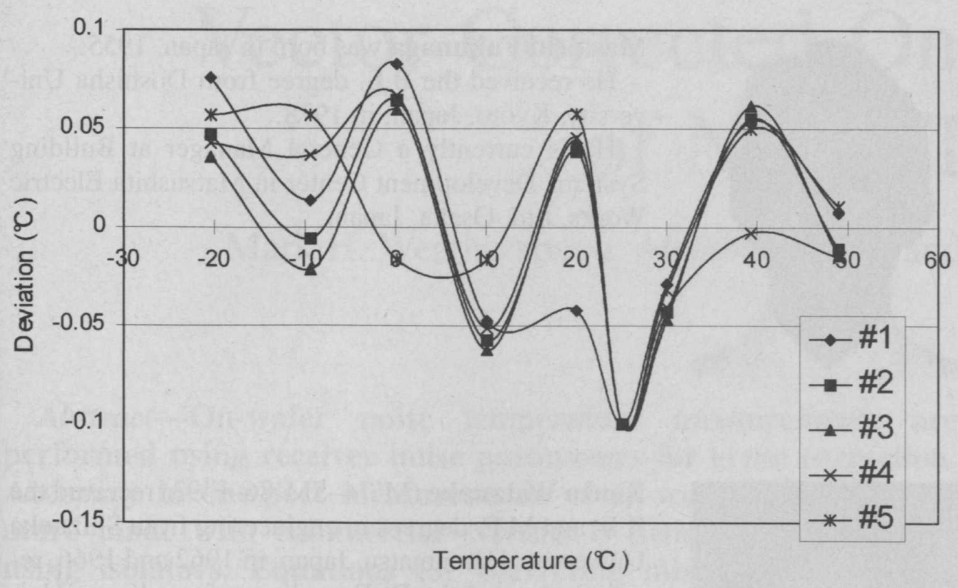

Fig. 7. Temperature measurement accuracy.

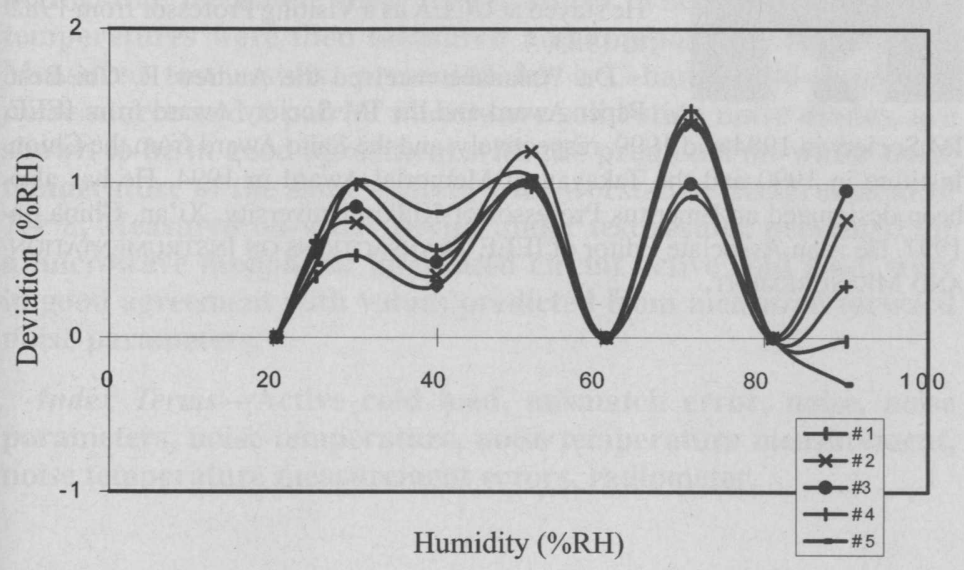

Fig. 8. RH measurement accuracy.

is $3.3 \mathrm{~V}$. The power dissipation is maximum when the STIM is communicating with clients and is $40 \mathrm{~mW}$.

The measurement accuracy of the prototype monitor has been evaluated by using a temperature- and $\mathrm{RH}$-controlled chamber. A commercial dew indicator has been used as a reference. The temperature measurement accuracy of the dew indicator is $\pm 0.1^{\circ} \mathrm{C}$. $\mathrm{RH}$ measurement accuracy is $\pm 1 \% \mathrm{RH}$ at $40 \% \mathrm{RH}$ and degrades to $\pm 2 \% \mathrm{RH}$ at $90 \% \mathrm{RH}$. The probe of the dew indicator is placed in close proximity to the prototype monitor to reduce error due to inhomogeneity to minimum. Figs. 7 and 8 show the measured results of five monitors numbered \#1 to \#5 sampled from 50 prototypes. In each figure, the abscissa indicates the reading of the dew indicator and the ordinate the difference between readings of the prototype monitor and the dew indicator. The discrepancies are within $\pm 0.1^{\circ} \mathrm{C}$ and $\pm 1.5 \% \mathrm{RH}$. Taking the propagation of errors into account, the uncertainties of prototype monitors are estimated to be $\pm 0.14{ }^{\circ} \mathrm{C}$ over the temperature range from $-20^{\circ} \mathrm{C}$ to $50{ }^{\circ} \mathrm{C}$ and $\pm 2.5 \% \mathrm{RH}$ over the range from $20 \% \mathrm{RH}$ to $90 \%$ $\mathrm{RH}$. The drift of the thermistor measured over $1,000 \mathrm{~h}$ when converted to temperature is within $0.25^{\circ} \mathrm{C}$, and that of the $\mathrm{RH}$ sensor aged at $40{ }^{\circ} \mathrm{C}, 90 \% \mathrm{RH}$ for ten days is within $1 \% \mathrm{RH}$ over $12000 \mathrm{~h}$. These performances are quite satisfactory for environment monitoring and thermal mapping.

Fig. 9 shows a web page made by Java Applet to display the measured temperature and RH. The display confirms that the prototype monitor is networked successfully to the Internet.

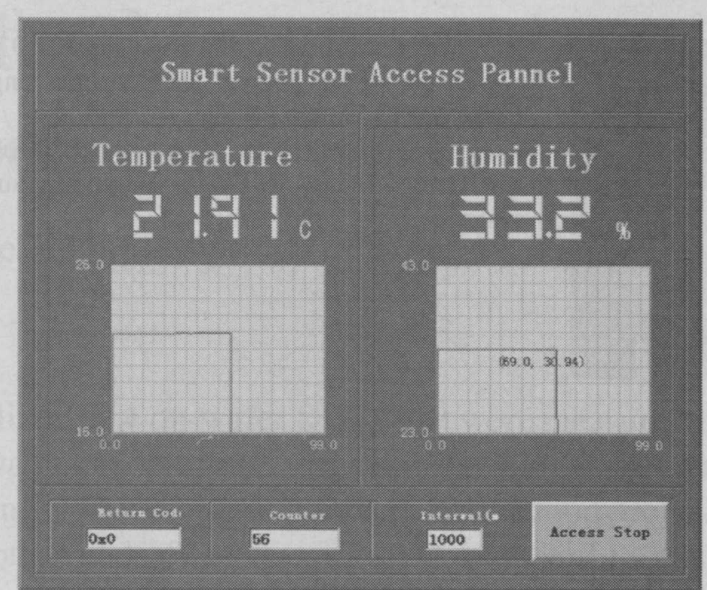

Fig. 9. An example of web page displaying the measured temperature and RH.

\section{CONCLUSION}

A temperature and $\mathrm{RH}$ monitor was developed successfully for the thermal environment assessment. The STIM designed based on the IEEE 1451.2 standard has allowed the online calibration and global networking. The prototype monitors have demonstrated such distinct features as low cost, high accuracy, low power consumption, easy installation, and easy networking. These distinct features made possible by the ratiometric signal conditioning, online calibration, and hierarchical network structure allow the monitor to find wide applicability in global assessment of thermal environments. It is also quite useful for thermal mapping of working space to save energy for air conditioning.

\section{REFERENCES}

[1] S Chen, K. Watanabe, M. Nakayama, M. Kanehori, H. Okayama, Y. Ito, and E. Kushime, "A low-cost, compact indoor thermal environment monitor," in Proc. IEEE Instrum. Meas. Technol. Conf., May 2000, pp. 290-294.

[2] K. Lee, "IEEE 1451: A standard in support of smart transducer networking," in Proc. IEEE Instrum. Meas. Technol. Conf., May 2000, pp. $525-528$.

[3] K. Lee, B. Schneeman, and D. Richard, "Distributed measurement and control based on the IEEE 1451 smart transducer interface standards," in Proc. IEEE Instrum. Meas. Technol. Conf., May 1999, pp. 608-613.

[4] H. Shibata, M. Ito, M. Asakursa, and K. Watanabe, "A digital hygrometer using a polyimide film relative humidity sensor," IEEE Trans. Instrum. Meas., vol. 45, no. 2, pp. 564-569, Apr. 1996.

[5] M. Bertocco, F. Ferraris, C. Offelli, and M. Parvis, "A client-server architecture for distributed measurement systems," IEEE Trans. Instrum. Meas., vol. 47, no. 5, pp. 1143-1148, Oct. 1998.

[6] M. A. Stegawshi and R. Schaumann, "A new virtual-instrumentationbased experimenting environment for undergraduate laboratories with application in research and manufacturing," IEEE Trans. Instrum. Meas., vol. 47, no. 6, pp. 1503-1506, Dec. 1998.

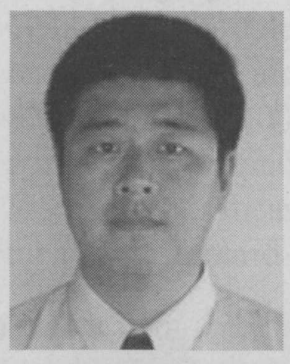

Yanfeng Wang (M'04) received the B.E. and the M.E. degrees in engineering from the Xidian University, China, in 1997 and 2001, respectively, and the Ph.D. degree from Shizuoka University, Japan, in 2004.

His current research interest is on the smart sensor signal process based on the wide area network. 


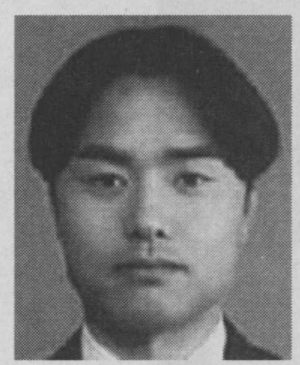

Makoto Nishikawa was born in Japan, 1974. He received the M.Sc. degree in electronics engineering from Mie University, Japan, in 1999.

$\mathrm{He}$ is currently with Matsushita Electric Works, Ltd, Osaka, Japan, developing a building automation system.

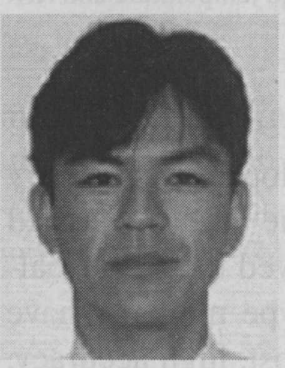

Ryuichi Maeda was born in Japan in 1967. He received the M.Sc. degree in system engineering from Hiroshima University, Japan, in 1992.

$\mathrm{He}$ is currently with Matsushita Electric Works, Ltd, Osaka, Japan, developing a building automation system.

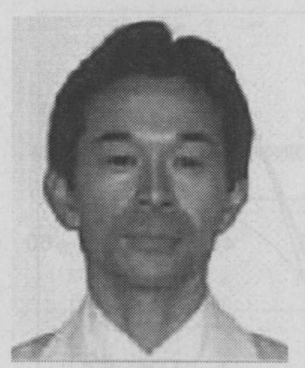

Masaichi Fukunaga was born in Japan, 1955.

He received the B.E. degree from Doshisha University, Kyoto, Japan, in 1978.

$\mathrm{He}$ is currently a General Manager at Building Systems Development Center in Matsushita Electric Works, Ltd, Osaka, Japan.

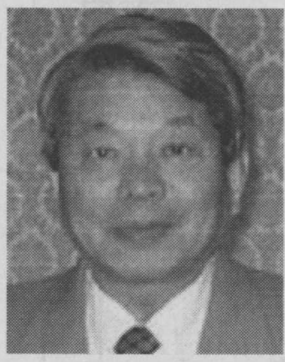

Kenzo Watanabe (M'74-SM'86-F'93) received the B.E. and M.E. degrees in engineering from Shizuoka University, Hamamatsu, Japan, in 1962 and 1966, respectively, and the Dr.Eng. degree from Kyoto University, Kyoto, Japan, in 1976.

$\mathrm{He}$ is currently a Professor and a director of the Research Institute of Electronics, Shizuoka University. He stayed at UCLA as a Visiting Professor from 1982 to 1983 .

Dr. Watanabe received the Andrew R. Chi Best Paper Award and the IM Society Award from IEEE IM Society in 1984 and 1999, respectively, and the Saito Award from the Chion Institute in 1990 and the Takayanagi Memorial Award in 1994. He has also been designated an Emeritus Professor of Xidian University, Xi' an, China, in 1997. He is an Associate Editor of IEEE TRANSACTIONS ON INSTRUMENTATION AND MEASUREMENT. 